\title{
Exploration and correlation analysis of changes in Krebs von den Lungen-6 levels in COVID-19 patients with different types in China
}

\author{
Mingshan Xue ${ }^{1, \S}$, Peiyan Zheng ${ }^{1, \S}$, Xiqing Bian ${ }^{2, \S}$, Zhifeng Huang ${ }^{1}$, Huimin Huang ${ }^{1}$, \\ Yifeng Zeng ${ }^{1}$, Haisheng $\mathrm{Hu}^{1}$, Xiaoqing Liu ${ }^{1}$, Luqian Zhou ${ }^{1}$, Baoqing Sun ${ }^{1, *}$, Jian-lin $\mathbf{W u}^{2, *}$, \\ Nanshan Zhong ${ }^{1, *}$
}

${ }^{1}$ State Key Laboratory of Respiratory Disease, National Clinical Research Center for Respiratory Disease, Guangzhou Institute of Respiratory Health, First Affiliated Hospital of Guangzhou Medical University, Guangzhou, China;

${ }^{2}$ State Key Laboratory of Quality Research in Chinese Medicine, Macau Institute for Applied Research in Medicine and Health, Macau University of Science and Technology, Macao, China.

SUMMARY This study aimed to determine the clinical significance of Krebs von den Lungen-6 (KL-6) in patients with COVID-19, so as to find a marker with high sensitivity, specificity and easy detection to evaluate the lung injury and inflammation of COVID-19. Sixty-three COVID-19 patients and 43 non-COVID-19 patients with similar clinical phenotypes and/or imaging findings were enrolled to test the levels of KL-6 using chemiluminescent immunoassay. In addition, the blood gas, imaging and lymphocyte factors tests were collected from all participants. The data was finally analyzed using multivariate statistical analysis. The results showed KL-6 levels in COVID-19 patients were higher than those in non-COVID-19 patients $(P<0.001)$. Moreover, the KL-6 levels in severe and critically severe patients were significantly upregulated compared with patients with mild and common type $(P<0.05)$. Meanwhile, the imaging evaluation showed a significant correlation between KL-6 and pulmonary lesion area $(P<0.05)$. KL-6 was also found to be significantly correlated with oxygenation index and oxygen partial pressure difference of alveolar artery $\left(\mathrm{PA}-\mathrm{aDO}_{2}\right)($ Both $P<0.01)$. In conclusion, KL-6 could be an indicator to evaluate the progression of COVID-19, which is parallel to the level of lung injury and inflammation in patients. Moreover, it can also reflect the pulmonary ventilation function.

Keywords COVID-19, lung, Krebs von den Lungen-6 (KL-6)

\section{Introduction}

The 2019 novel coronavirus pneumonia (COVID-19) was initially identified in Wuhan, China in December 2019, and emerged as a global pandemic, which affected greater than 3,500,000 people globally, including more than 110,000 deaths by May, 2020, and rising. Based on its partial homology with SARS-CoV-2 virus, the novel coronavirus of this pandemic was named COVID-19 by the World Health Organization (WHO) (1) and confirmed to be the seventh member of the coronavirus family of beta-coronavirus (2). COVID-19 can cause symptoms of acute nonspecific respiratory infection, such as fever, dry cough, shortness of breath, as well as many pulmonary manifestations, such as diarrhea and muscle soreness; in addition, many patients present no clinical symptoms, which will cause the virus to spread widely (2-4). Many severe patients can develop septic shock, acute respiratory distress syndrome, disseminated intravascular coagulation (DIC), and multiple organ failure during progressive progression (5). Severe COVID-19 cases might lead to death when they suffer from cardiovascular disease, diabetes, liver failure, and heart failure $(6,7)$. Therefore, it is urgent to find an easily, simple and effective diagnosis approach for COVID-19 to reduce mortality.

Current diagnostic approaches for COVID-19 include nucleic acid detection, chest $\mathrm{CT}$, epidemiological history and clinical manifestations (8-10). However, nucleic acid detection is time consuming and an incorrect sample collection may lead to false-positive results. Additionally, the specificity of $\mathrm{CT}$ is low and the cost is high. Moreover, in clinical practice, the detection standard varied partly with rapidly growing awareness 
of COVID-19 (7,8). Hence, timely and accurate diagnosis of COVID-19 is still important for detection and therapy of patients.

Krebs von den Lungen-6 (KL-6), a high molecular weight mucinous glycoprotein, is more highly expressed in injured or regenerating epithelial cells than in normal epithelial cells $(11,12)$. KL-6 plays an important role in pulmonary inflammatory damage, mainly due to its ability to specifically identify the function of type 1 alveolar epithelial cells, thus as a biomarker to predict risk of illness or death of pneumonia patients (13). Angiotensin-converting enzyme 2 (ACE2) mainly exists in type 1 alveolar epithelium (14). Receptor binding domain (RBD) glycoprotein, the functional receptor of COVID-19, could closely bind to ACE2, thus resulting in significant reduction of the number of viruses for infecting cells (15). Hence, the pathogenesis of COVID-19 might be related to KL-6. In the present study, we explored the changes of KL-6 levels in COVID-19 and non-COVID-19 patients, aiming to investigate the pathogenesis and auxiliary diagnostic markers of COVID-19, as well as further analyze its transmission characteristics. The flow chart of the experiment is shown in Figure 1.

\section{Materials and Methods}

\subsection{Study design and patients}

A total of 63 COVID-19 cases were involved, which were collected and collated by the First Affiliated Hospital of Guangzhou Medical University between February 1, 2020 and March 15, 2020. According to the standard of COVID-19 medical protocol issued by national health commission, the patients were divided into two groups: a mild and common type group $(N=$ $30)$, as well as a severe and critically severe type group $(N=33)$, by evaluation of results of clinical symptoms, imaging and blood gas (standard atmospheric pressure). However, due to lack of follow-up data in part of the COVID-19 patients, some patients were excluded in the process of collating information. Finally, 6 patients with mild or normal type and 15 patients with severe or critical type were included in the longitudinal followup analysis. In addition, 43 gender and age-matched confirmed non-COVID-19 patients with similar clinical phenotypes and/or imaging findings were enrolled. The clinical parameters of all participants were collected and summarized in Table 1. This study was Approved by the ethics committee of First Affiliated Hospital of Guangzhou Medical University (Ethics number 202077).

\subsection{Diagnosis}

The patients were recruited according to guidelines of diagnosis and treatment of COVID-19 including clinical characteristics and typical epidemiological history. Inclusion criteria were as follows: $i$ ) COVID-19 was positive after PCR detection of nucleic acid in respiratory or blood samples; $i$ ) The sequence of virus genes in respiratory or blood samples showed a high homology to COVID-19. Meanwhile, we also referred to the diagnostic and grading criteria of Daniel et al. 2020 (16), Jin et al. 2020 (17) and Corman et al. 2020 (1). Briefly, the clinical manifestations were consistent with fever and/or respiratory symptoms, imaging features of COVID-19 infection, normal or decreased white blood cells, and normal or decreased lymphocyte count.

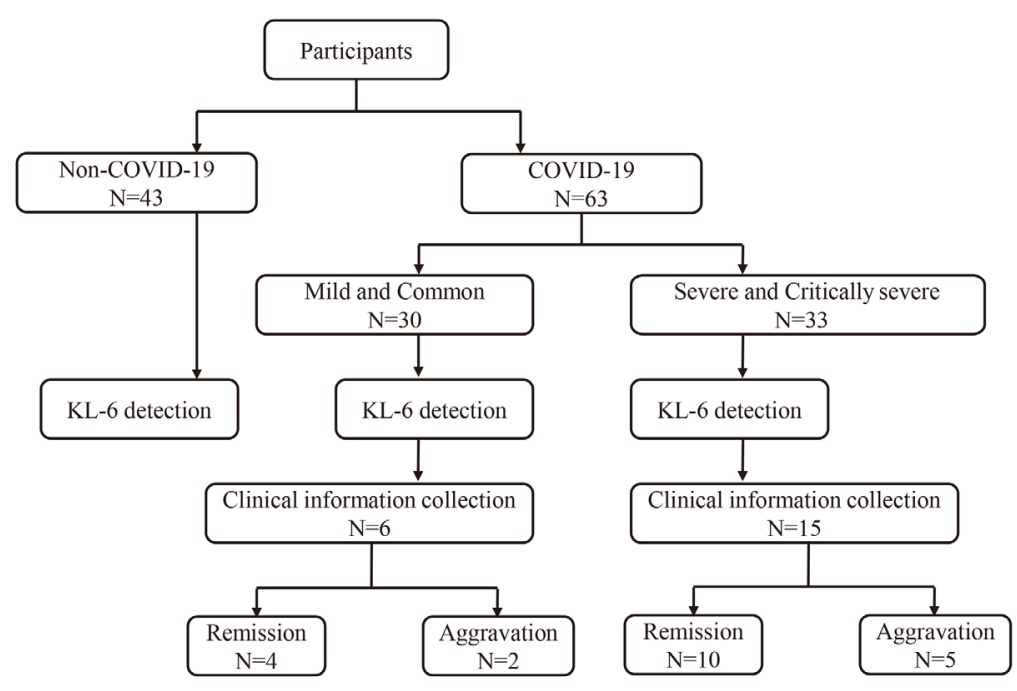

Figure 1. The flow chart of the experiment. 
Table 1. The clinicopathologic characteristics of participants

\begin{tabular}{|c|c|c|c|c|}
\hline \multirow{2}{*}{ Variables } & \multirow{2}{*}{ Non-COVID-19 } & \multicolumn{2}{|c|}{ Non-COVID-19 } & \multirow{2}{*}{$P$ value } \\
\hline & & Mild and Common & Severe and Critically Severe & \\
\hline$N$ & 43 & 6 & 15 & \\
\hline Age, years & $51.70 \pm 3.27$ & $55.00 \pm 18.84$ & $57.20 \pm 14.25$ & 0.015 \\
\hline Gender, male/female & $30 / 13$ & $2 / 4$ & $12 / 3$ & 0.098 \\
\hline KL-6, U/mL & $173.9 \pm 63.40$ & $241.2 \pm 207.90$ & $676.6 \pm 506.70$ & 0.001 \\
\hline $\mathrm{CRP}, \mathrm{mg} / \mathrm{L}$ & - & $4.77 \pm 3.59$ & $8.38 \pm 6.77$ & 0.027 \\
\hline $\mathrm{LDH}, \mathrm{U} / \mathrm{L}$ & - & $411.00 \pm 359.40$ & $492.30 \pm 718.40$ & 0.151 \\
\hline Blood creatinine, umol/L & - & $79.77 \pm 22.93$ & $90.32 \pm 44.48$ & 0.159 \\
\hline ALT, U/L & - & $41.35 \pm 45.43$ & $55.06 \pm 50.20$ & 0.121 \\
\hline $\mathrm{AST}, \mathrm{U} / \mathrm{L}$ & - & $42.07 \pm 26.28$ & $171.10 \pm 856.40$ & 0.014 \\
\hline Oxygenation index & - & $3.99 \pm 1.60$ & $2.21 \pm 2.11$ & 0.001 \\
\hline $\mathrm{PA}-\mathrm{aO}_{2}$ & - & $145.30 \pm 69.50$ & $219.40 \pm 125.10$ & 0.003 \\
\hline \multicolumn{5}{|l|}{ Venous blood cell analysis } \\
\hline White blood cell, $10^{9} / \mathrm{L}$ & - & $5.16 \pm 1.68$ & $9.29 \pm 3.88$ & 0.001 \\
\hline Neutrophil count, $10^{9} / \mathrm{L}$ & - & $3.48 \pm 1.57$ & $7.40 \pm 3.72$ & 0.001 \\
\hline Lymphocyte count, $10^{9} / \mathrm{L}$ & - & $1.07 \pm 0.34$ & $0.97 \pm 0.61$ & 0.001 \\
\hline Eosinophil count, $10^{9} / \mathrm{L}$ & - & $0.11 \pm 0.06$ & $0.22 \pm 0.28$ & 0.037 \\
\hline Red blood cell, $10^{12} / \mathrm{L}$ & - & $3.66 \pm 0.55$ & $2.97 \pm 0.62$ & 0.001 \\
\hline Platelet count, $10^{9} / \mathrm{L}$ & - & $273.5 \pm 104.0$ & $178.40 \pm 80.30$ & 0.001 \\
\hline \multicolumn{5}{|l|}{ Electrolyte } \\
\hline $\mathrm{K}, \mathrm{mmol} / \mathrm{L}$ & - & $3.86 \pm 0.31$ & $4.40 \pm 5.88$ & 0.001 \\
\hline $\mathrm{Na}, \mathrm{mmol} / \mathrm{L}$ & - & $138.40 \pm 1.78$ & $141.10 \pm 6.29$ & 0.001 \\
\hline $\mathrm{CI}, \mathrm{mmol} / \mathrm{L}$ & - & $104.4 \pm 2.77$ & $106.4 \pm 8.04$ & 0.030 \\
\hline $\mathrm{Ca}, \mathrm{mmol} / \mathrm{L}$ & - & $2.23 \pm 0.10$ & $2.25 \pm 0.18$ & 0.432 \\
\hline
\end{tabular}

ALT, Alanine transaminase; AST, Glutamic oxalacetic transaminase; CRP, C-reactive protein; KL-6, Krebs von den Lungen-6; LDH, Lactate dehydrogenase; $\mathrm{PA}-\mathrm{aO}_{2}$, Partial pressure difference of oxygen in alveolar air artery.

\subsection{Sample collection and preservation}

$5 \mathrm{~mL}$ venous blood samples were collected, divided into individual tubes and stored at $0-4^{\circ} \mathrm{C}$ for inspection, and the other blood was centrifuged at 3,000 rpm for $30 \mathrm{~min}$ at room temperature to obtain serum. Urine samples were collected, stored at $0-4^{\circ} \mathrm{C}$ and measured within 24 hours. Feces samples ( $5 \mathrm{~g}$ ) were collected and stored at room temperature until analysis.

\subsection{KL-6 detection}

The levels of KL-6 in serum were measured using chemiluminescence immunity (KAESER 1000) according to the manufacturers' protocol.

\subsection{Imaging evaluation}

Due to the condition limitation of severe patients and for the purpose of dynamic observation of the lesion change level, we also included the results of DR results. The appearance, size, and location of pulmonary infection lesions in DR and CT reports were both evaluated. Coronal plane was used for evaluation of DR and CT. CT was also assessed from four planes: subclavicular, aortic arch, hilum, and superior diaphragm. According to the grading standards reported by Chung et al. (18) and Lei et al. (19), > 50\% intrapulmonary lesion progression from 24 to 48 hours was classified as the severe classification.

\subsection{Statistical analysis}

R software (Bell Laboratories Version 4.0.0), SPSS 22.0 software (SPSS Inc., Chicago, IL, USA) and GraphPad Prism 5.0 (G San Diego, CA, US) were used for statistical analysis. Comparisons in three groups or between two groups were made by ANOVA or Student's $t$-test. $P$ value $<0.05$ was considered to be statistically significant.

\section{Results}

\subsection{Participants characteristics}

As shown in Table 1, the levels of KL-6 in patients with COVID-19 were significantly higher than those in non-COVID-19 type $(P<0.001)$. Moreover, the levels of AST in patients with severe and critically severe were upregulated in comparison with those in patients with mild and common type, and the difference of AST levels between the two groups was significant $(P$ $<0.05)$. Additionally, no electrolyte disturbance was observed in COVID-19 patients.

\subsection{Changes of KL-6 level in COVID-19 patients with different types}

To detect the potential roles of KL- 6 in progression of COVID-19 disease, we assessed the change of KL-6 levels in COVID-19 patients with different types. As 
illustrated in Figure 2A, the KL-6 level of COVID-19 patients was significantly higher than that of nonCOVID-19 controls, and the overall levels of KL-6 in severe and critically severe patients were significantly higher than that in mild and common patients (both $P<0.01)$. The overall levels of KL-6 in patients with COVID-19 after remission showed a decreasing trend. During hospitalization, the levels of KL-6 rose briefly due to changes in the condition, but gradually decreased with the overall improvement of the condition in the later period; moreover, the length of hospitalization for mild and common type of patients was relatively short (Figure 2B). However, not all COVID-19 patients improved during hospitalization. For example, in this study, a total of 5 critically severe patients have aggravated conditions during hospitalization. According to Figure 2C, KL-6 levels in 2 of them showed a significant upward trend in the first 16 days. Taken together, the KL-6 level of the patients with aggravation of the disease was stable and increased; however, the change trend of KL- 6 in patients with mild and common type was basically stable due to their mild condition. In this study, 2 cases of mild and common type were aggravated, among which 1 case showed a significant upward trend of KL-6 level.

\subsection{Imaging analysis of pulmonary lobe involvement}

To further clarify the clinical significance of KL-6 in COVID-19 patients, the phenotypic data in imaging reports of COVID-19 patients were investigated.
There was no significant change of lesion size in mild and common group, however, we observed a significant reduction in lesions of severe and critically severe stages of remission. Additionally, There was a significant correlation between KL-6 and pulmonary lesion area. ( $r=-0.14, P<0.05$; Table 2$)$.

\subsection{Inflammatory index analysis}

Inflammatory index analysis showed that lymphocytes of severe and critically severe patients have a temporary

Table 2. Imaging findings of the COVID-19 patient's lungs

\begin{tabular}{|c|c|c|}
\hline Variables & $\begin{array}{c}\text { Mild and } \\
\text { Common, } N\end{array}$ & $\begin{array}{c}\text { Severe and } \\
\text { Critically Severe, } N\end{array}$ \\
\hline
\end{tabular}

\begin{tabular}{lcc}
\hline Lung involvement area & & \\
$0 \%$ & 0 & 0 \\
$1-25 \%$ & 6 & 0 \\
$26-50 \%$ & 0 & 1 \\
$51-75 \%$ & 0 & 11 \\
$76-100 \%$ & 0 & 3 \\
Morphologic & & \\
Patchy shadows & $6(100.0)$ & $15(100.0)$ \\
Consolidations & $4(66.7)$ & $9(60.0)$ \\
Pleural thickening & 0 & $3(20.0)$ \\
Bronchiolectasis & $1(16.7)$ & $4(26.7)$ \\
Atelectasis & 0 & $1(6.7)$ \\
Tuberosis & $1(16.7)$ & $4(26.7)$ \\
Hydrothorax & 0 & $7(46.7)$ \\
Mediastinal lymphadenectasis & 0 & $4(26.7)$ \\
Emphysema & 0 & $6(40.0)$ \\
Pulmonary fibrosis & 0 & $1(6.7)$ \\
\hline
\end{tabular}

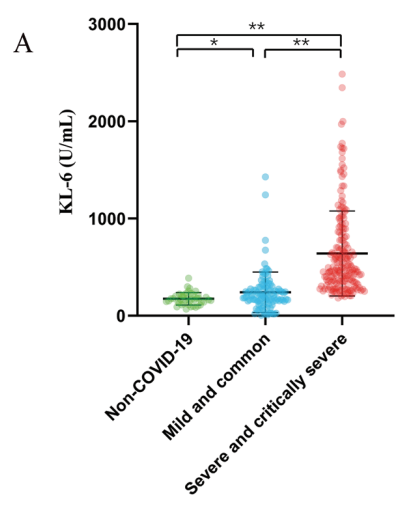

C
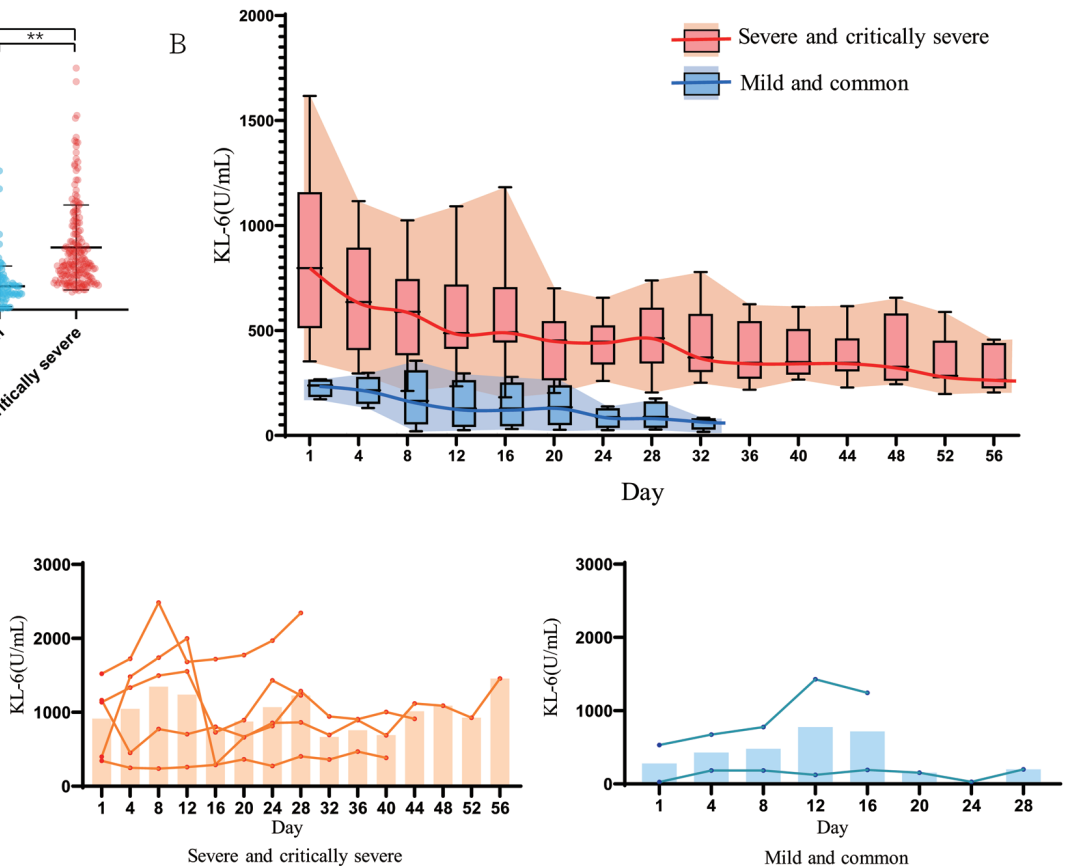

Figure 2. KL-6 level in COVID-19 patients with different types. (A) The differences in non- COVID-19 controls group, mild and common patients group and severe and critically severe patients group. (B) Changes in KL-6 levels in remission patients with COVID-19; (C) Changes in KL-6 level in patients with aggravating COVID-19. KL-6, Krebs von den Lungen-6. 

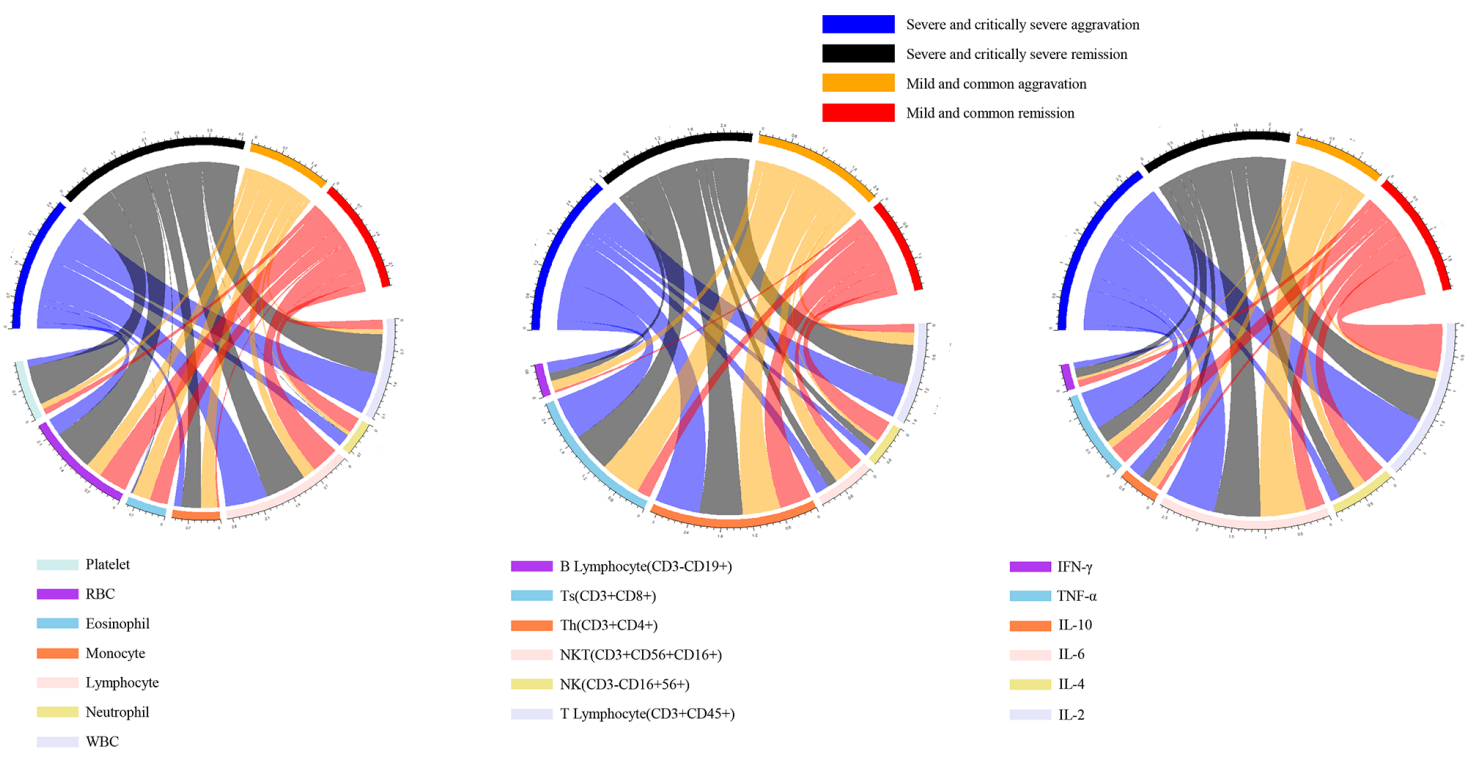

Figure 3. Correlation analysis of KL-6 level with leukocyte, lymphocyte subsets and inflammatory factors in COVID-19 patients. IFN, Interferon; IL, Interleukin; NK, Natural killer; NKT, Natural killer T lymphocyte; Th, Helper T lymphocyte; TNF, Tumor necrosis factor; Ts, Suppressor T lymphocyte.

decline at the time of admission, and then increase to normal with remission of the disease. The levels of $\mathrm{T}$ lymphocytes $(\mathrm{CD} 3+)$, helper $\mathrm{T}$ lymphocytes $(\mathrm{CD} 3+\mathrm{CD} 4+)$ and inhibitory $\mathrm{T}$ lymphocytes $(\mathrm{CD} 3+\mathrm{CD} 8+)$ increased gradually with progression of the COVID-19 patients, while there was no significant changes in B lymphocytes (CD3-CD19+) and NK cells $(\mathrm{CD} 3-\mathrm{CD} 16+56+)$. Next, the correlation analysis of leukocyte, lymphocyte subsets and inflammatory cytokines with KL-6 levels was conducted. The results revealed that there was a significant correlation between the levels of KL-6 and T lymphocyte $(\mathrm{CD} 3+\mathrm{CD} 45+)$ in severe and critically severe patients $(r=-0.24, P<$ 0.05 ; Figure 3$)$. Moreover, the Ts $(\mathrm{CD} 3+\mathrm{CD} 8+)$ and $\mathrm{Th}$ $(\mathrm{CD} 3+\mathrm{CD} 4+)$ lymphocyte subsets were both closely related to KL-6 levels in all COVID-19 patients $(r=$ -0.19 and -0.25 , both $P<0.05$ ). Additionally, IL-6 and IL-10 were significantly correlated with KL-6 levels, respectively $(r=0.38$ and 0.19 , both $P<0.05)$.

3.5. Correlation analysis of blood gas and KL-6 in patients

According to Figure 4, 81.5\% of severe and critically severe patients had an oxygenation index $\left(\mathrm{PaO}_{2} / \mathrm{FiO}_{2}\right)$ of less than 3.00 within 5 days of admission. In general, KL-6 was significantly correlated with $\mathrm{CO}_{2}$ partial pressure, arterial alveolar blood oxygen partial pressure difference and oxygenation index $\left(\mathrm{PaO}_{2} / \mathrm{FiO}_{2}\right)$ under normal atmospheric pressure in COVID-19 patients $(r$ $=0.19,0.48$ and -0.45 , all $P<0.01$ ).

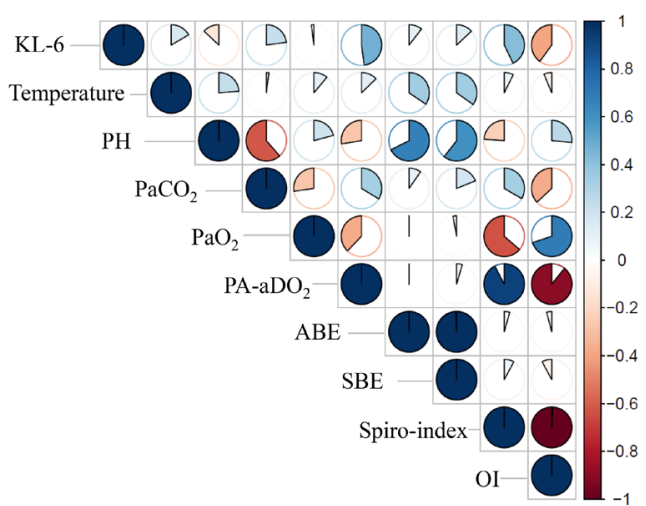

Figure 4. Correlation analysis of blood gas and KL-6 in patients. PA-aDO ${ }_{2}$ : Alveolar-arterial oxygen differential; ABE: Actual alkali excess; SBE: Standard alkali excess; Spiro-index: Respiratory Index; OI: Oxygenation index.

\section{Discussion}

According to the follow-up investigation of in-patient conditions, the levels of KL-6 were different with change of COVID-19 patients' condition. Moreover, the expression level of KL-6 was significantly correlated with oxygenation index and arterial alveolar oxygen partial pressure difference. At the same time, it was also consistent with the change of the pulmonary lesion area. In particular, in patients with COVID-19, no obvious electrolyte abnormality was observed.

In this study, the levels of KL-6 in COVID-19 patients was found to be higher than in non-COVID-19 patients with similar symptoms. During the course 
of the disease, the levels of KL-6 were parallel to the changes of the disease, especially in severe and critical severe patients. Actually, with aggravation of the disease, a large amount of viral replication infiltration could destroy the alveolar epithelium, cause damage to the basement membrane, increase pulmonary vascular permeability, and result in localized pulmonary edema (13). At the same time, a clear membrane is formed, which leads to symptoms of chest tightness and shortness of breath (20). In our study, the levels of KL-6 were consistent with the severity of the disease and the progressive changes in the course of the disease. Therefore, we speculated that it could reflect the damage of alveolar epithelium in patients with COVID-19.

Besides, the lymphocyte count of some patients with severe and critically severe COVID-19 was lower than normal, and gradually increased to normal with the recovery of immune function, which was consistent with the characteristics of other viral infections. Through the detection of lymphocyte subsets, we found that the total number of CD3 $+\mathrm{T}$ lymphocytes increased with the progress of the disease, and at the same time, auxiliary T lymphocytes (Th CD3+CD4+) and inhibitory $\mathrm{T}$ lymphocytes (Ts $\mathrm{CD} 3+\mathrm{CD} 8+$ ) also increased to regulate the immune function of the body. No significant changes were observed in CD3-CD19+ B lymphocytes and CD3-CD16+56+ of NK cells. It was found that KL-6 was significantly correlated with changes of cytokine IL-6 in the whole course of disease. IL-6 was one of the cytokines which could lead to fever and increase of alveolar epithelial exudation (21). Early rapid replication of the virus may lead to a large amount of airway and alveolar epithelial and endothelial cell apoptosis, and induce release of a large amount of pro-inflammatory cytokines and chemokines (20). We speculated that the level of KL-6 was correlated with some $\mathrm{T}$ lymphocytes and inflammatory factors and could reflect the level of lung inflammation. Oxygenation index $\left(\mathrm{PaO}_{2} / \mathrm{FiO}_{2}\right)$ in $81.5 \%$ of patients with severe and critical type was less than 3.00 on day 5 and rose to normal with remission. COVID-19 can cause lung interstitial injury. In addition, the partial pressure difference of the alveolar artery is also related to the severity and improvement of the disease. KL-6 is significantly correlated with the above two indicators. Under the action of infection and inflammation, interstitial edema and destruction of pulmonary vascular endothelium will occur in the lungs, and the respiratory membrane ventilation efficiency will be significantly reduced. In our study about imaging report, the pulmonary interstitial fibrosis induced by severe viral pneumonia was also found to have an influence on lung ventilation function and abnormal blood gas indicators. KL-6 is one of the indicators for assessing the degree of pulmonary interstitial fibrosis and is related to the prognosis of the disease. In addition, KL- 6 was related to the oxygenation index and the differential pressure of alveolar arterial oxygen and could reflect the pulmonary ventilation function in our study. Therefore, KL-6 could be used as a key indicator to evaluate the degree of damage of alveolar epithelial cells.

The study showed that lung lobe involvement was higher in severe and critically severe patients than in mild and common patients. Moreover, in the aggravation stage, the progress of lung lesion area in COVID-19 patients was significantly correlated with the level of KL-6. Hydrothorax, pleural thickening, and Mediastinal lymphadenectasis due to heavy infection are also more common in severe and critically severe patients. From the perspective of vision, patients with severe and critically severe COVID-19 had a wider lung involvement area, which was consistent with the degree of lung damage.

Compared to detecting biomarkers in COVID-19 by nucleic acid detection and chest CT, measurement of the levels of KL-6 were rapid, sensitive, and inexpensive. Several studies suggest that KL-6 is associated with lung cancer $(22,23)$. However, no articles have been reported about the relationship of KL-6 with COVID-19. Therefore, KL- 6 could be regarded as a novel biomarker for COVID-19. In our study, the levels of KL-6 were closely related to IL-6, IL-10, $\mathrm{PaCO}_{2}, \mathrm{PaO}_{2} / \mathrm{FiO}_{2}$ and pulmonary lesion area. These results suggested that KL-6 is a useful prognostic indicator for COVID-19.

Although promising results were obtained, this study also has some limitations. For example, the number of patients was not sufficient to perform a valid statistical analysis. To investigate the significance of KL-6 expression, a larger number of patients with COVID-19 is required for a prospective study.

\section{Conclusion}

In summary, KL-6 can be used as an auxiliary evaluation index of lung injury due to COVID-19, and can reflect lung ventilation function and oxygen intake level of patients. In addition, KL-6 could be used as a new biomarker in the future for screening and evaluating COVID-19 by virtue of its high sensitivity, specificity and easy detection.

Funding: This study was supported by Zhejiang University special scientific research fund for COVID-19 prevention and control (2020XGZX040).

Conflict of Interest: The authors declare that they have no competing interests.

\section{References}

1. Corman VM, Landt O, Kaiser M. et al. Detection of 2019 novel coronavirus (2019-nCoV) by real-time RT-PCR. Euro Surveill. 2020; 25:2000045.

2. Zhu Y, Li C, Chen L. et al. A novel human coronavirus 
OC43 genotype detected in mainland China. Emerg Microbes Infect. 2018; 7:173.

3. Yang XL, Hu B, Wang B, Wang MN, Zhang Q, Zhang W, Wu LJ, Ge XY, Zhang YZ, Daszak P, Wang LF, Shi ZL. Isolation and Characterization of a Novel Bat Coronavirus Closely Related to the Direct Progenitor of Severe Acute Respiratory Syndrome Coronavirus. J Virol. 2015; 90:3253-3256.

4. Wang C, Horby PW, Hayden FG, Gao GF. A novel coronavirus outbreak of global health concern. Lancet. 2020; 395:470-473

5. Hui DS, E IA, Madani TA, Ntoumi F, Kock R, Dar O, Ippolito G, McHugh TD, Memish ZA, Drosten C, Zumla A, Petersen E. The continuing 2019-nCoV epidemic threat of novel coronaviruses to global health - The latest 2019 novel coronavirus outbreak in Wuhan, China. Int J Infect Dis. 2020; 91:264-266.

6. Malik YS, Sircar S, Bhat S, Sharun K, Dhama K, Dadar M, Tiwari R, Chaicumpa W. Emerging novel coronavirus (2019-nCoV)-current scenario, evolutionary perspective based on genome analysis and recent developments. Vet Q. 2020; 40:68-76.

7. $\mathrm{Wu} Z$ Z, McGoogan JM. Characteristics of and Important Lessons From the Coronavirus Disease 2019 (COVID-19) Outbreak in China: Summary of a Report of 72314 Cases From the Chinese Center for Disease Control and Prevention. JAMA. 2020; doi: 10.1001/jama.2020.2648.

8. To KK, Tsang OT, Chik-Yan Yip C. et al. Consistent detection of 2019 novel coronavirus in saliva. Clin Infect Dis. 2020; ciaa149. doi: 10.1093/cid/ciaa149.

9. Zu ZY, Jiang MD, Xu PP, Chen W, Ni QQ, Lu GM, Zhang LJ. Coronavirus Disease 2019 (COVID-19): A Perspective from China. Radiology. 2020; 200490. doi: 10.1148/radiol.2020200490.

10. Sharfstein JM, Becker SJ, Mello MM. Diagnostic Testing for the Novel Coronavirus. JAMA. 2020; doi: 10.1001/ jama.2020.3864.

11. Kohno N, Awaya Y, Oyama T, Yamakido M, Akiyama M, Inoue Y, Yokoyama A, Hamada H, Fujioka S, Hiwada K. KL-6, a Mucin-like Glycoprotein, in Bronchoalveolar Lavage Fluid from Patients with Interstitial Lung Disease. Am Rev Respir Dis. 1993; 148:637-642.

12. Ishizaka A, Matsuda $\mathrm{T}$, Albertine $\mathrm{KH}$, Koh H, Tasaka S, Hasegawa N, Kohno N, Kotani T, Morisaki H, Takeda J, Nakamura M, Fang X, Martin TR, Matthay MA, Hashimoto S. Elevation of KL-6, a lung epithelial cell marker, in plasma and epithelial lining fluid in acute respiratory distress syndrome. Am J Physiol Lung Cell Mol Physiol. 2004; 286:L1088-1094.

13. $\mathrm{Hu} \mathrm{C}$, Wu C, Yang E, Huang H, Xu D, Hou Y, Zhao J, Li M, Xu Z, Zeng X, Wang Q. Serum KL-6 is associated with the severity of interstitial lung disease in Chinese patients with polymyositis and dermatomyositis. Clin Rheumatol. 2019; 38:2181-2187.

14. Chen Y, Guo Y, Pan Y, Zhao ZJ. Structure analysis of the receptor binding of 2019-nCoV. Biochem Biophys Res Commun. 2020; 525:135-140.

15. Hamming I, Timens W, Bulthuis ML, Lely AT, Navis
$\mathrm{G}$, van Goor H. Tissue distribution of ACE2 protein, the functional receptor for SARS coronavirus. A first step in understanding SARS pathogenesis. J Pathol. 2004; 203:631-637.

16. Chu DKW, Pan Y, Cheng SMS, Hui KPY, Krishnan P, Liu Y, Ng DYM, Wan CKC, Yang P, Wang Q, Peiris M, Poon LLM. Molecular Diagnosis of a Novel Coronavirus (2019-nCoV) Causing an Outbreak of Pneumonia. Clin Chem. 2020; 66:549-555.

17. Jin YH, Cai L, Cheng ZS. et al. A rapid advice guideline for the diagnosis and treatment of 2019 novel coronavirus (2019-nCoV) infected pneumonia (standard version). Mil Med Res. 2020; 7:4.

18. Chung M, Bernheim A, Mei X, Zhang N, Huang M, Zeng X, Cui J, Xu W, Yang Y, Fayad ZA, Jacobi A, Li K, Li S, Shan H. CT Imaging Features of 2019 Novel Coronavirus (2019-nCoV). Radiology. 2020; 295:202-207.

19. Lei J, Li J, Li X, Qi X. CT Imaging of the 2019 Novel Coronavirus (2019-nCoV) Pneumonia. Radiology. 2020; 295:18.

20. Fu Y, Cheng Y, Wu Y. Understanding SARS-CoV-2Mediated Inflammatory Responses: From Mechanisms to Potential Therapeutic Tools. Virol Sin. 2020; 1-6.

21. Tanaka T, Narazaki M, Kishimoto T. Interleukin (IL-6) Immunotherapy. Cold Spring Harb Perspect Biol. 2018; 10:a028456.

22. Tanaka S, Hattori N, Ishikawa N, Shoda H, Takano A, Nishino R, Okada M, Arihiro K, Inai K, Hamada HJijoc. Krebs von den Lungen-6 (KL-6) is a prognostic biomarker in patients with surgically resected nonsmall cell lung cancer. Int J Cancer. 2012; 130:377-387.

23. Takanashi S, Nishina N, Nakazawa M, Kaneko Y, Takeuchi TJR. Usefulness of serum Krebs von den Lungen- 6 for the management of myositis-associated interstitial lung disease. Rheumatology (Oxford). 2019; 58:1034-1039.

Received May 29, 2020; Revised June 8, 2020; Accepted June 11, 2020.

${ }^{\S}$ These authors contributed equally to this work.

*Address correspondence to:

Baoqing Sun and Nanshan Zhong, State Key Laboratory of Respiratory Disease, National Clinical Research Center for Respiratory Disease, Guangzhou Institute of Respiratory Health, First Affiliated Hospital of Guangzhou Medical University, Guangzhou 510120, China.

E-mail: sunbaoqing@vip.163.com (Sun BQ); nanshan@, vip.163.com (Zhong NS)

Jian-lin Wu, State Key Laboratory of Quality Research in Chinese Medicine, Macau Institute for Applied Research in Medicine and Health, Macau University of Science and Technology, Macao, China.

E-mail: jlwu@must.edu.mo

Released online in J-STAGE as advance publication June 21, 2020. 